\title{
Étude par spectroscopie Mössbauer du fer (III) dans des verres silicatés multicomposants d'intérêt géologique
}

\author{
P. Levitz $\left({ }^{*}\right)$, G. Calas $\left({ }^{* *}\right)$, D. Bonnin $(*)$ et A. P. Legrand $\left({ }^{*}\right)$ \\ (*) Laboratoire de Physique Quantique, ERA 676, 10, rue Vauquelin, 75231 Paris Cedex 05, France \\ $\left.{ }^{(* *}\right)$ Laboratoire de Minéralogie-Cristallographie, LA 09, Université Pierre-et-Marie-Curie, 75230 Paris Cedex 05, France
}

(Reçu le 15 novembre 1979, révisé le 22 janvier 1980, accepté le 28 janvier 1980)

\begin{abstract}
Résumé. - Cet article décrit la spectroscopie Mössbauer du Fe (III) dans des verres silicatés synthétiques d'intérêt géologique. La composition chimique de ces verres s'étend d'une composition basique, verres de diopside ou de basalte, à une composition acide, verres d'albite ou de granite. Une nouvelle méthode d'analyse des spectres Mössbauer contenant du Fe (II) et du Fe (III) est décrite. Celle-ci permet d'obtenir le spectre d'absorption Mössbauer du $\mathrm{Fe}$ (III) sans hypothèse sur la forme théorique de la raie comme l'ont fait d'autres auteurs. On trouve que le Fe (III) est en coordination 4. L'influence de la composition chimique est étudiée.
\end{abstract}

\begin{abstract}
This paper deals with Mössbauer absorption of Fe (III) in some synthetic silicate glasses which are interesting in geology. The chemical composition of these glasses spreads from a basic chemical composition as the diopside or basaltic glass to acid chemical composition as albite or granit glass. A new method of analysing the Mössbauer spectra containing Fe (II) and Fe (III) is described. This one permits to obtain the Mössbauer absorption of $\mathrm{Fe}$ (III) with no hypothesis on the theoretical shape of this line as usually used by other authors. Fe (III) is found in coordination 4 . The influence of the chemical composition is studied.
\end{abstract}

1. Introduction. - La compréhension des réactions d'oxydo-réduction dans les liquides silicatés est importante aussi bien en sidérurgie et dans l'industrie du verre que pour l'étude des processus magmatiques fondamentaux. Trois facteurs principaux influent sur les équilibres redox : la pression partielle d'oxygène, la température et la composition du liquide $[1,2]$. Parmi les ions à valences multiples, le fer est de loin le plus abondant, et son importance en géologie vient du fait que l'équilibre $\mathrm{Fe}$ (II)/Fe (III) tamponne les autres réactions d'oxydo-réduction et contrôle les activités de nombreuses phases minérales dans les magmas terrestres. D'autre part si le rôle modificateur du fer divalent dans les structures des liquides silicatés est admis actuellement, il n'en est pas de même du fer trivalent dont on connaît mal le comportement, soit modificateur soit formateur de réseau.

La spectroscopie Mössbauer a été largement utilisée dans la détermination du rapport $\mathrm{Fe}$ (II)/Fe (III), plus particulièrement dans les systèmes simples : $\mathrm{SiO}_{2}-\mathrm{Na}_{2} \mathrm{O}$ [3], $\mathrm{SiO}_{2}-\mathrm{CaO}-\mathrm{Fe}_{2} \mathrm{O}_{3}$ [4], $\mathrm{SiO}_{2}-\mathrm{CaO}-$ $\mathrm{Al}_{2} \mathrm{O}_{3}-\mathrm{Fe}_{2} \mathrm{O}_{3}$ [5], $\mathrm{SiO}_{2}-\mathrm{Na}_{2} \mathrm{O}-\mathrm{CaO}-\mathrm{Fe}_{2} \mathrm{O}_{3}$ [6], verres silicatés multicomposants [7] et verres boratés alcalins [8]. Ces études ont été faites sur des verres synthétisés généralement en conditions oxydantes ou légèrement réductrices et à température relativement basse, ce qui a pour effet de favoriser le fer (III) nettement prépondérant.

Nous nous sommes intéressés dans cette étude à des verres plus complexes, correspondant aux deux types principaux de magmas terrestres, basaltiques et grani- tiques. Les conditions rencontrées dans les systèmes naturels sont assez réductrices, typiquement au voisinage de l'équilibre $\mathrm{Ni} / \mathrm{NiO}$, d'où la nécessité d'une décomposition correcte du spectre Mössbauer pour bien séparer la contribution très importante du fer (II) du spectre global.

2. Conditions expérimentales. - Les synthèses des verres ont été réalisées à partir des mélanges d'oxydes préalablement chauffés en plusieurs étapes. Les quatre verres étudiés sont un verre de diopside, $\mathrm{CaMgSi}_{2} \mathrm{O}_{6}$, un verre d'albite, $\mathrm{NaAlSi}_{3} \mathrm{O}_{8}$, un verre de basalte $\left(47,9 \% \mathrm{SiO}_{2}, 26,46 \% \mathrm{Al}_{2} \mathrm{O}_{3}, 0,1 \% \mathrm{MnO}, 9,78 \% \mathrm{MgO}\right.$, $15,22 \% \mathrm{CaO}, 0,3 \% \mathrm{Na}_{2} \mathrm{O}$ ) et un verre de granite $\left(73,1 \% \quad \mathrm{SiO}_{2}, \quad 15,05 \% \quad \mathrm{Al}_{2} \mathrm{O}_{3}, \quad 0,92 \% \mathrm{MgO}\right.$, $2,07 \% \mathrm{CaO}, 3,62 \% \mathrm{Na}_{2} \mathrm{O}, \quad 4,27 \% \mathrm{~K}_{2} \mathrm{O}$ ). Les fusions ont été faites dans des fours à axe vertical, en atmosphère contrôlée par des mélanges gazeux $\mathrm{H}_{2}-\mathrm{CO}_{2}$ et $\mathrm{CO}-\mathrm{CO}_{2}$. La trempe est obtenue par chute dans l'eau et les équilibres mesurés dans le verre sont considérés comme représentatifs de ceux existant dans le liquide silicaté. Les potentiels d'oxygène déterminés à partir des compositions des gaz sont contrôlés à l'aide des équilibres $\mathrm{Fe} / \mathrm{FeO}$ et $\mathrm{Ni} / \mathrm{NiO}$ au voisinage desquels ont été faites les synthèses en atmosphère réductrice. Les capsules utilisées sont soit en platine pour les potentiels d'oxygène élevés, soit en molybdène, surtout au tampon $\mathrm{Fe} / \mathrm{FeO}$, à cause des pertes importantes en fer dans les capsules en platine à faible potentiel d'oxygène. Les liquides sont équilibrés avec l'atmosphère du four pendant 
des durées de 3 à $12 \mathrm{~h}$, suffisantes pour obtenir l'équilibre des états d'oxydation et pour éviter des pertes en alcalins importantes aux températures élevées (1500 ${ }^{\circ} \mathrm{C}$ typiquement). En vue des études par effet Mössbauer, les verres ont été dopés avec $1 \mathrm{mg}$ de ${ }^{57} \mathrm{Fe}_{2} \mathrm{O}_{3}$, ce qui correspond à $5 \%$ environ du poids total pour des capsules contenant en moyenne $20 \mathrm{mg}$ de produit. Cette valeur est couramment rencontrée dans les roches naturelles (typiquement entre 2 et $12 \%$ selon la composition).

Les verres synthétisés ont été broyés et déposés sur un porte-échantillon de $3 \mathrm{~cm}^{2}$. L' "épaisseur » de ces absorbants est de l'ordre de $0,3 \mathrm{mg} / \mathrm{cm}^{2} \mathrm{de}{ }^{57} \mathrm{Fe}$. La source utilisée est constituée de ${ }^{57} \mathrm{Co}$ diffusé dans une matrice de rhodium et a une activité de $10 \mathrm{mCi}$. Les spectres ont été enregistrés en mode triangulaire sur 400 canaux puis repliés autour du canal central, et l'étalonnage en vitesse a été fait à partir d'un spectre de fer métallique. Les déplacements isomériques sont donnés par rapport au fer naturel $\alpha$.

3. Méthode d'étude du fer (III). - De façon générale, les spectres Mössbauer des verres oxydés présentent trois maxima caractéristiques de la superposition des doublets quadrupolaires du fer (II) et du fer (III). Le recouvrement est maximal dans la région de vitesse nulle.

Pour l'étude de ces spectres, le premier type de traitement possible est l'ajustement de la courbe expérimentale par une somme de doublets lorentziens symétriques par une méthode d'optimisation non linéaire de type Fletcher-Powell [9]. Cette technique, couramment utilisée, pose un certain nombre de problèmes. En premier lieu elle peut aboutir à une décomposition numériquement acceptable mais ne présentant pas un caractère d'unicité suffisant. D'autre part elle implique des hypothèses sur la forme et la symétrie des courbes d'absorption qui, dans les verres, n'ont pas de justifications physiques évidentes. Le choix d'un doublet lorentzien symétrique pour décrire le comportement du fer (II) $[4,5,6]$ est en contradiction avec l'assymétrie caractéristique des spectres d'absorption du fer ferreux dans les verres et avec leur caractère non lorentzien très marqué $[10,11]$.

A cette méthode d'analyse des verres oxydés, nous préférons une technique d'ajustement tenant mieux compte du comportement spécifique du fer divalent dans les verres.

3.1 DESCRIPTION DE LA MÉTHODE. - Cette méthode proche de celle utilisée par Varret et Naudin [7] pour l'étude de verres silicatés à faible teneur en fer ( $<1000 \mathrm{ppm}$ ) consiste à comparer le spectre Mössbauer d'un verre contenant les deux types de valence $\mathrm{Fe}$ (II) et $\mathrm{Fe}$ (III) au même matériau équilibré dans des conditions très réductrices (tampon $\mathrm{Fe}-\mathrm{FeO}$ ) où seul n'est présent que le $\mathrm{Fe}$ (II).

(1) Le raisonnement sera fait ici sur les spectres en absorption.
Dans ce dernier cas, l'absence de Fe (III) est aisément vérifiable par $\mathrm{RPE}$, où la raie à $g=4,3$, caractéristique de cet état de valence en milieu amorphe, est alors inexistante.

Le spectre Mössbauer du verre oxydé peut s'écrire $\left({ }^{1}\right)$

$$
\mathrm{S}(\mathrm{v})=\mathrm{Y} 3(\mathrm{v})+\mathrm{Y} 2(\mathrm{v})
$$

où Y3(v) est la contribution quadrupolaire du fer (III) et $\mathrm{Y} 2(\mathrm{v})$ celle du fer (II). Appelons R(v) le spectre d'absorption du même verre réduit.

Normalisons $R(v)$ à la hauteur maximale de $S(v)$. Dans ces conditions :

et

$$
\begin{gathered}
\mathrm{Y} 2(\mathrm{v})=\lambda \mathrm{R}(\mathrm{v}) \text { avec } 0<\lambda<1 \\
\mathrm{~S}(\mathrm{v})=\mathrm{Y} 3(\mathrm{v})+\lambda \mathrm{R}(\mathrm{v})
\end{gathered}
$$

pour connaître Y3(v) il nous faut définir $\lambda$. Pour cela nous considérerons que dans la zone $(+1,9 \mathrm{~mm} / \mathrm{s}$, $+4 \mathrm{~mm} / \mathrm{s})$, l'absorption Mössbauer du verre oxydé provient essentiellement du fer (II).

Comme montré en figure 1 une bonne estimation de $\lambda$ devra donc permettre de faire coïncider au mieux la portion de courbe $C D$ attachée à $R(v)$, à la partie $\mathrm{AB}$ du spectre $\mathrm{S}(\mathrm{v})$.

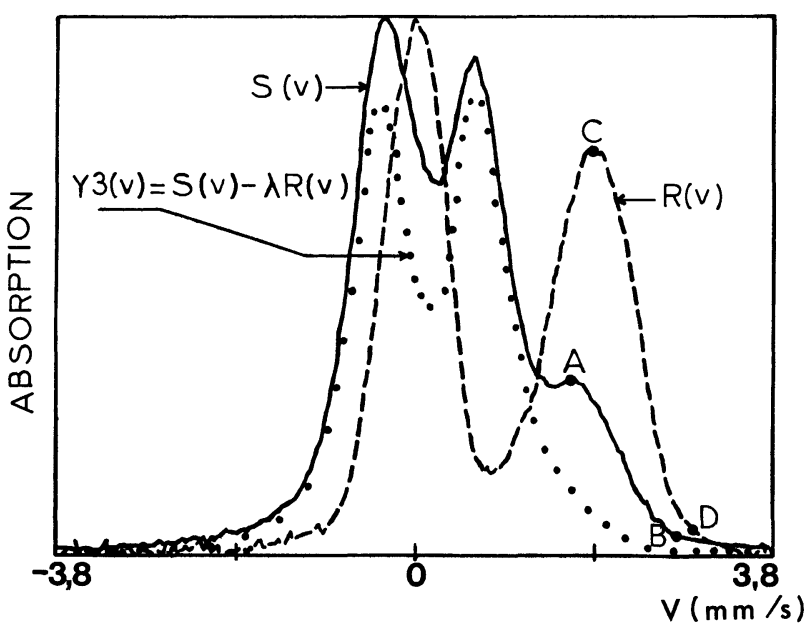

Fig. 1. - Méthode de décomposition d'un spectre Mössbauer d'un verre contenant du Fe (II) et du Fe (III).

[Method of resolving a Mössbauer spectrum in $\mathrm{Fe}$ (II) and $\mathrm{Fe}$ (III) containing glass.]

Dans ces conditions la courbe Y3(v) obtenue par simple soustraction doit présenter des faibles valeurs et une décroissance monotone dans la zone $(+2 \mathrm{~mm} / \mathrm{s},+4 \mathrm{~mm} / \mathrm{s})$. Ce fait atteste d'une bonne élimination de l'absorption imputable au fer (II). Cette contrainte définit alors une valeur minimale $\lambda_{\text {min }}$ admissible de $\lambda$. D'autre part Y3(v) étant une courbe d'absorption, est essentiellement positive. Ceci impose une valeur maximale $\lambda_{\max }$ de $\lambda$ à ne pas dépasser. Dans l'intervalle $\left[\lambda_{\min }, \lambda_{\max }\right]$ il est alors possible de choisir selon un critère visuel, une valeur de $\lambda$ qui fasse coïncider au mieux la branche $C D d u$ 
Tableau I. - Conditions de synthèse des verres étudiés et caractéristiques des spectres du fer trivalent obtenus par la méthode de soustraction.

\begin{tabular}{|c|c|c|c|c|c|c|c|c|c|}
\hline Numéro & Composition & $\begin{array}{c}\text { Température } \\
\text { d'équilibration }\end{array}$ & $\begin{array}{l}\text { Atmosphère } \\
\text { du four }\left(\mathrm{PO}_{2}\right)\end{array}$ & $\lambda$ & $\begin{array}{c}\text { Déplacement } \\
\text { isomérique } \\
(\mathrm{mm} / \mathrm{s}) \\
\mathrm{Fe}(\mathrm{III})\end{array}$ & $\begin{array}{c}\text { Ecart } \\
\text { quadrupolaire } \\
(\mathrm{mm} / \mathrm{s}) \\
\mathrm{Fe}(\mathrm{III})\end{array}$ & $\begin{array}{c}\text { Largeur } \\
\text { apparente } \\
\text { à mi-hauteur } \\
(\mathrm{mm} / \mathrm{s}) \\
\mathrm{Fe} \text { (III) }\end{array}$ & $\frac{\mathrm{Fe} \text { (III) }}{\mathrm{Fe} \text { (II) }}$ & Références \\
\hline 1 & basalte & $1420^{\circ} \mathrm{C}$ & $10^{-9,7}$ & & & & & $\sim 0$ & $\begin{array}{l}\text { Référence de } \\
\text { Fe (II) pour (2) } \\
\text { et (3) }\end{array}$ \\
\hline 2 & basalte & $1440^{\circ} \mathrm{C}$ & $10^{-5,1}$ & 0,92 & 0,28 & 1,34 & 0,80 & 0,30 & \\
\hline 3 & basalte & $1420^{\circ} \mathrm{C}$ & $10^{-0,7}$ & 0,46 & 0,29 & 1,33 & 0,76 & 2,05 & \\
\hline 4. & albite & $1480^{\circ} \mathrm{C}$ & $10^{-8,5}$ & & & & & $\sim 0$ & $\begin{array}{l}\text { Référence de } \\
\text { Fe (II) pour (5) } \\
\text { et (6) }\end{array}$ \\
\hline 5 & albite & $1550^{\circ} \mathrm{C}$ & $10^{-0,7}$ & 0,65 & 0,26 & 1,22 & 0,88 & 1,05 & \\
\hline 6 & granite & $1550^{\circ} \mathrm{C}$ & $10^{-0,7}$ & 0,73 & 0,26 & 1,35 & 0,73 & 0,95 & \\
\hline 7 & diopside & $1410^{\circ} \mathrm{C}$ & $10^{-0.7}$ & 0,60 & 0,27 & 1,25 & 0,98 & 1 & \\
\hline 8 & diopside & $1410^{\circ} \mathrm{C}$ & $10^{-9.7}$ & & & & & $\sim 0$ & $\begin{array}{l}\text { Référence de } \\
\text { Fer (II) pour (7) }\end{array}$ \\
\hline
\end{tabular}

spectre du verre réduit à la branche $\mathrm{AB}$ du spectre du verre oxydé.

3.2 Mise EN ceUvRe De LA MÉTHODE ET Résultats OBTENUS. - De façon générale la valeur optimale du paramètre $\lambda$ peut être obtenue avec une incertitude de $5 \%$. Le rapport des surfaces associées aux doublets respectifs du fer (II) et du fer (III) donne alors le rapport $\mathrm{Fe}$ (II)/Fe (III). Il faudrait théoriquement corriger ce rapport en le divisant par celui des fractions résonnantes correspondantes que nous n'avons pas déterminées. En pratique il est raisonnable de penser que la correction sera faible. Les principaux résultats

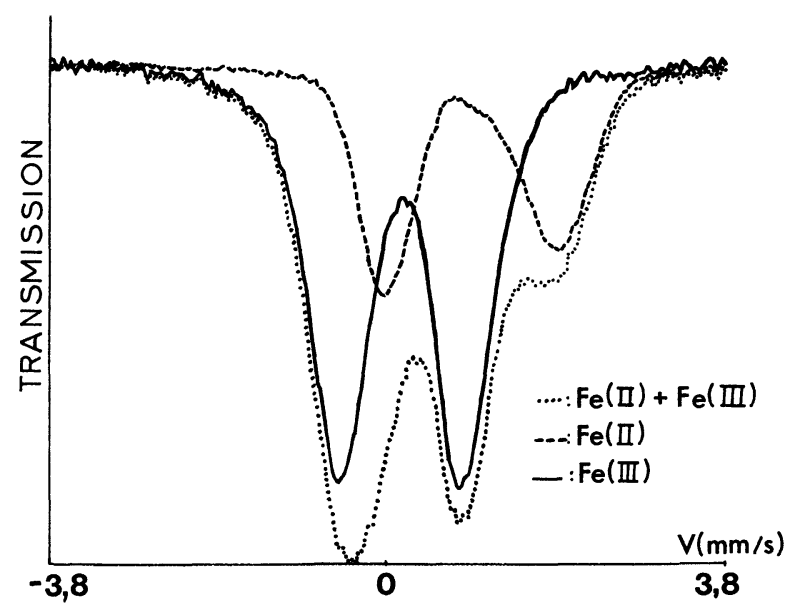

Fig. 2. - Décomposition d'un spectre de verre basaltique (verre 3).

[Resolution of a basaltic glass spectrum (glass 3).] obtenus sont résumés dans le tableau I les spectres décomposés sont représentés sur les figures 2 à 4 .

De façon générale, le spectre du fer (III) obtenu après soustraction est un doublet quadrupolaire symétrique, très élargi dont le déplacement isomérique moyen diminue légèrement en passant des verres basaltiques aux verres granitiques.

Un tel résultat est à rapprocher de ceux obtenus sur des verres très oxydés $[5,8]$. On peut donc penser que la validité de la méthode utilisée repose sur le fait que le spectre du Fe (II) dans un verre oxydé est très semblable à celui obtenu sur le même verre réduit, ce qui indique que l'environnement local autour du fer (II) ne change pas avec la fugacité d'oxygène.

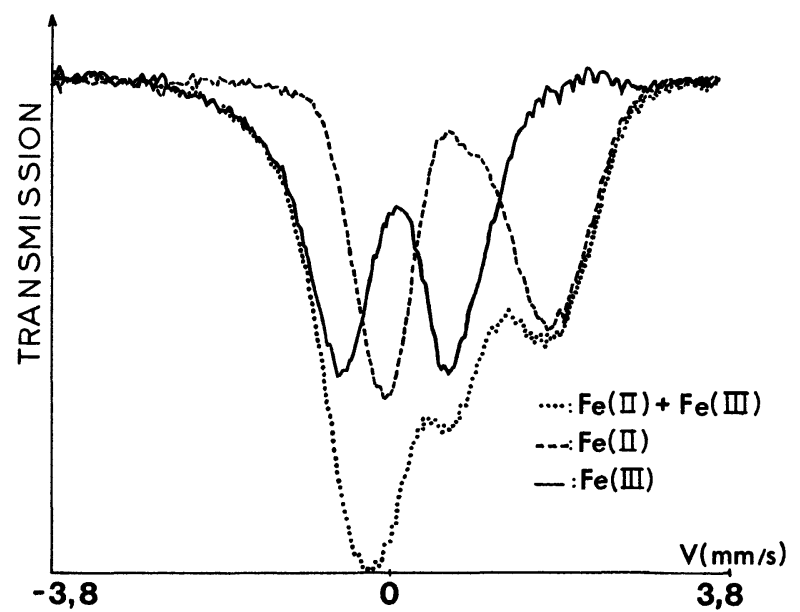

Fig. 3. - Décomposition d'un spectre de verre d'albite (verre 5). [Resolution of an albitic glass spectrum (glass 5).] 


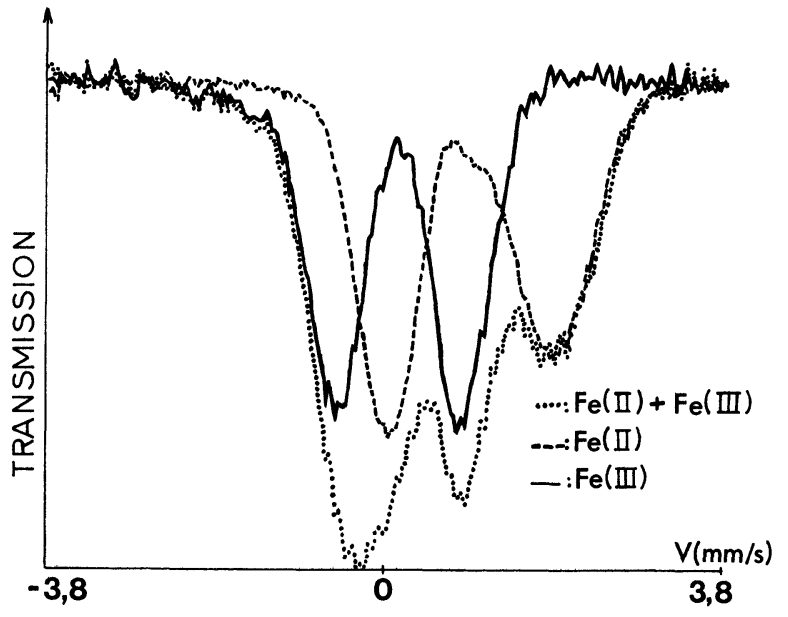

Fig. 4. - Décomposition d'un spectre de verre granitique (verre 6).

[Resolution of a granitic glass spectrum (glass 6).]

4. Evolution du rapport Fe (II)/Fe (III). - L'équilibre des deux valences du fer dans un liquide silicaté peut s'écrire selon la réaction générale

$$
\mathrm{Fe}(\mathrm{II})+1 / 4 \mathrm{O}_{2(\mathbf{z})} \leftrightarrows \mathrm{Fe}(\mathrm{III})+1 / 2 \mathrm{O}^{2-}
$$

dont la constante d'équilibre est :

$$
K=\frac{(\mathrm{Fe}(\mathrm{III}))\left(\mathrm{O}^{2-}\right)^{1 / 2}}{(\mathrm{Fe}(\mathrm{II}))\left(\mathrm{fO}_{2}\right)^{1 / 4}}
$$

les parenthèses indiquent les activités.

Cette constante d'équilibre est une fonction de la température et de la composition du liquide. Pour une même composition, le rapport des concentrations de chaque valence varie donc comme la puissance $1 / 4$ de la fugacité d'oxygène. Ceci est bien vérifié dans le cas des verres basaltiques par exemple (cf. Tableau I), ce qui montre que la méthode de décomposition utilisée donne des résultats cohérents.

D'autre part, on constate que les liquides basaltiques sont plus oxydants que les trois autres compositions étudiées. Ceci n'est pas surprenant dans le cas des liquides granitiques ou à composition d'albite, car il s'agit de bains fortement polymérisés où l'activité des ions oxydes $\left(\mathrm{O}^{2-}\right)$ est faible. La différence est plus surprenante dans le cas des bains à composition diopside qui sont beaucoup moins polymérisés $(\mathrm{Si} / \mathrm{O}=0,33)$. Ce résultat est à rapprocher de celui obtenu par Lauer et Schreiber [12], qui trouvent en RPE peu de différences entre des verres à composition de feldspath et de diopside alors qu'un changement dans la nature du cation modificateur change beaucoup le rapport $\mathrm{Fe}$ (II)/Fe (III) au voisinage du rapport $\mathrm{Si} / \mathrm{O}=0,33$. Une telle évolution pourrait être envisagée lors du passage d'une composition diopside à une composition basaltique et amène à la conclusion que les activités des ions oxydes $\left(\mathrm{O}^{2-}\right)$ mesurées par un couple redox n'ont qu'une signification relative : l'étude de l'équilibre Eu (II)/Eu (III) dans les mêmes compositions montre au contraire qu'un verre basaltique est légèrement plus réducteur qu'un verre de diopside et qu'un verre d'albite est beaucoup plus réducteur que ce dernier [12].

5. Interprétation des paramètres Mössbauer du fer (III). - Les doublets de fer (III) obtenus à l'aide de notre méthode de soustraction de spectres sont comparables à ceux obtenus par les autres auteurs en conditions plus oxydantes [3-6]. Cependant, même dans le cas où la quantité de fer divalent est faible, l'utilisation d'un doublet lorentzien symétrique pour représenter son absorption introduit une perturbation sur la composante à faible vitesse interférant avec le spectre du fer (III), et ceci d'autant plus que l'atmosphère de synthèse est plus réductrice. D'autre part, Pargamin et al. (5) et Lévy et al. [4, 6], ont décomposé ce doublet de fer (III) en deux doublets lorentziens symétriques, de déplacements isomériques sensiblement identiques et d'écarts quadrupolaires différents. Le doublet d'écart quadrupolaire le plus élevé $(>1,10 \mathrm{~mm} / \mathrm{s})$ est associé à une coordination de 4 et le plus faible $(<1 \mathrm{~mm} / \mathrm{s})$ à une coordination de 6 . Ce type de décomposition amène les auteurs à observer des variations relatives des deux doublets en fonction de la composition du bain, de la température et de la pression partielle d'oxygène, ce qui dans les deux derniers cas traduit, au moins en partie, les perturbations dues à la variation de la concentration en fer (II) lorsque l'absorption de celui-ci est représentée par un simple doublet lorentzien symétrique.

Sur un plan plus général, l'attribution d'une coordination du fer (III) à l'aide des spectres Mössbauer demande une certaine prudence. L'écart quadrupolaire, par exemple, n'est pas généralement considéré comme caractéristique d'une coordination [13]. On constate au contraire que le déplacement isomérique dans les composés cristallisés est nettement différent entre les deux coordinations, 4 et 6 du fer (III), il en est ainsi par exemple dans le cas des ferri-diopsides [14].

Deux paramètres importants $[15,16]$ influent sur le déplacement isomérique, le nombre de coordination et la distance fer-oxygène. Une diminution de la distance métal-ligand induit un abaissement du déplacement isomérique par l'augmentation du caractère covalent de la liaison fer-oxygène. Le passage d'une coordination 6 à une coordination 4 définit une évolution du même type dont l'une des causes est identique. On sait en effet que les distances $\mathrm{Fe}$ (III) - ligand en coordination tétraédrique sont abaissées en général de 5 à $7 \%$ par rapport à celles rencontrées en coordination octaédrique. Il en est ainsi par exemple dans le cas du YIG [17] $\left(\mathrm{Y}_{2} \mathrm{Fe}_{2}\left(\mathrm{FeO}_{4}\right)_{3}\right)$ où le fer (III) en site $\mathrm{d}$ (tétraédrique) possède une distance $\mathrm{Fe}-\mathrm{O}$ de $1,88 \AA$ et un déplacement isomérique de $0,22 \mathrm{~mm} / \mathrm{s}$ alors que le même ion en site a (octaédrique) se caractérise par une distance $\mathrm{Fe}-\mathrm{O}$ de $2 \AA$ et un déplacement isomérique de $0,41 \mathrm{~mm} / \mathrm{s}$. 
Confirmant ce type d'approche Kurkjian [3] a d'ailleurs défini une échelle de variation du déplacement isomérique du fer (III) permettant de séparer les deux types d'environnements dans les milieux amorphes silicatés et phosphatés. La coordination 4 est définie par une valeur du déplacement isomérique comprise entre $0,20 \mathrm{~mm} / \mathrm{s}$ et $0,30 \mathrm{~mm} / \mathrm{s}$ (réf. fer $\alpha$ ) et la coordination 6 par une valeur supérieure ou égale à $0,40 \mathrm{~mm} / \mathrm{s}$.

Il semble donc difficile d'accepter le type de décomposition adopté par Lévy et al. $[4,6]$ et Pargamin et al. [5], à moins d'admettre la possibilité d'un octaèdre d'insertion du fer (III) très comprimé. Nous considérons plutôt que la symétrie caractéristique des spectres d'absorption du fer (III) ainsi que le déplacement isomérique moyen trouvé sont significatifs d'un site d'insertion tétracoordonné légèrement relaxé. La valeur élevée de l'écart quadrupolaire moyen trouvée de façon systématique dans les différents verres étudiés est significative d'une distortion importante par rapport à la symétrie cubique. De telles valeurs ne sont pas exceptionnelles et peuvent être rencontrées dans un certain nombre de clinopyroxènes cristallisés [14].

On note enfin une légère différence entre les paramètres des spectres des différents verres étudiés : le déplacement isomérique diminue faiblement en passant d'un verre basaltique à un verre granitique. Ceci peut être dû à un changement dans la polarisation des oxygènes qui ligandent le fer (III), en relation avec la forte baisse en éléments "modificateurs" entre un bain basaltique et un bain granitique. Ces éléments induisant une forte polarisation du cortège électronique des oxygènes ont pour conséquence une diminution des recouvrements orbitaux entre fer (III) et l'oxygène, induisant une augmentation du déplacement. isomérique. Une autre hypothèse peut être également une légère diminution des distances $\mathrm{Fe}$ (III)-O en passant d'un bain basaltique à un bain granitique.

6. Conclusion. - Le traitement présenté ici, permet de caractériser l'ion fer (III) dans des verres étudiés en spectrométrie Mössbauer, par un doublet quadrupolaire symétrique. La valeur du déplacement isomérique moyen qui s'y rattache, ainsi que sa faible fluctuation incite à conclure que cet ion est tétracoordonné.

Le spectre Mössbauer du fer (II) n'a été ici utilisé que d'un point de vue purement empirique. Il est cependant intéressant de s'interroger sur sa forme dissymétrique qui est caractéristique de cet état de valence dans les verres $[7,10]$.

Pour ce faire, il est nécessaire de comparer les conclusions qu'apportent trois types d'expériences successives : la spectrométrie Mössbauer, la spectrométrie d'absorption optique et l'EXAFS. Cette discussion comparative est menée par ailleurs [11, $21,22]$; elle permet de caractériser l'environnement du fer (II), par la présence simultanée de deux types de sites distribués géométriquement et de coordinations différentes : un site tétracoordonné et un site hexacoordonné.

Une analyse plus détaillée de l'environnement du fer (III) par trois spectroscopies précédemment citées se présente de façon différente. D'une part l'interprétation des spectres Mössbauer semble moins délicate que pour l'ion ferreux. D'autre part les spectres d'adsorption optique du fer (III) sont rarement résolus dans les verres synthétisés à haute température à cause d'une faible bande de transfert de charge fer-oxygène dans l'ultra-violet.

Il serait alors intéressant de comparer les résultats présentés ici à ceux que fournirait l'EXAFS de façon à obtenir des informations sur l'influence de la nature chimique du bain silicaté sur l'évolution de la covalence de la liaison fer (III)-O.

\section{Bibliographie}

[1] Wong, J. et ANGell, C. A., Glass structure by spectroscopy (Marcel Dekker, New York) 1976.

[2] BAMFORD, C. R., Colour generation and control in glass (Elsevier) 1977.

[3] Kurkian, C. R. et Sigety, E. A., Phys. Chem. Glasses 9 (1968) 73.

[4] Levy, R. A., in "Amorphous Magnetism II", ed. R. A. Levy et R. Hasegawa (Plenum, New York) p. 613.

[5] Pargamin, L., Lupis, C. M. P. et Flinn, P. A., Metallurgical Trans 3 (1972) 2093.

[6] Levy, R. A., Lupis, C. M. P. et Fuinn, P. A., Phys. Chem. Glasses 17 (1976) 94.

[7] Varret, F. et Naudin, F., Revue Phys. Appl. 14 (1979) 613.

[8] Raman, T. et Rao, G. N., J. Non Cryst. Sol. 29 (1978) 85.

[9] Fletscher, R. et Powell, M. J. D., The Computer Journal 6 (1963) 163.

[10] Mao, H. K. et Bell, P. M., Carnegie Inst. Yearbook 72 (1973) 629.

[11] Levitz, P., Bonnin, D., Calas, G. D. et Legrand, A. P., J. Phys. E (à paraître).
[12] Lauer, H. V. et SChreiber, H. D., in « Rare-Earths in modern science and technology », ed. G. J. Mc Carthy et J. J. Rhyne (Plenum, New York), p. 283.

[13] Coey, J. M. D., J. Physique Colloq. 35 (1974) C6-89.

[14] VIRGO, D., Carnegie Inst. Washington Yearbook 71 (1972) 534.

[15] Sawatzky, G. A. et Van der Woude, F., J. Physique Colloq. 35 (1974) C6-47.

[16] Simanek, E. et Sroubek, Z., Phys. Rev. 163 (1967) 275.

[17] Nicholson, W. J. et Burns, G., Phys. Rev. 133A (1964) 1568.

[18] Loveridge, D. et Parke, S., Phys. Chem. Glasses 12 (1971) 19.

[19] Raoux, D., Petiau, J., Bondot, P., Calas, G., Fontaine, A., Lagarde, P., Levitz, P., Loupias, G., Sadoc, A., Revue Phys. Appl. (à paraître).

[20] Communication orale.

[21] Calas, G., Levitz, P., Loupias, G., Petiau, J. et Bondot, P., Revue Phys. Appl. (à paraître).

[22] Levitz, P., Thèse 3e cycle Paris (1979). 